\title{
Examination of the use of needle biopsy to perform laparoscopic surgery safely on uterine smooth muscle tumors
}

\author{
MAKOTO MURAKAMI ${ }^{1}$, TOMOYUKI ICHIMURA ${ }^{1}$, MARI KASAI $^{1}$, MAKIKO MATSUDA $^{2}$, \\ NAOKI KAWAMURA ${ }^{3}$, TAKESHI FUKUDA ${ }^{1}$ and TOSHIYUKI SUMI ${ }^{1}$ \\ ${ }^{1}$ Department of Gynecology, Osaka City University, Abeno-ku, Osaka 5458586; ${ }^{2}$ Department of Obstetrics and Gynecology, \\ Osaka City Sumiyoshi Hospital, Suminoe-ku, Osaka 5590012; ${ }^{3}$ Department of Gynecology, \\ Osaka City General Hospital, Miyakojima-ku, Osaka 5340021, Japan
}

Received May 6, 2016; Accepted December 20, 2016

DOI: $10.3892 / 01.2018 .8425$

\begin{abstract}
The warning statement issued by the United States Food and Drug Administration against the use of laparoscopic power morcellators prompted a discussion about the methods of preoperative diagnosis of uterine myometrial lesions. Since 1994, transcervical needle biopsies have been performed to differentiate between uterine leiomyomas and leiomyosarcomas. Needle biopsies are also useful for performing laparoscopic surgery on uterine smooth muscle tumors with histopathological safety. In the present study, data from hematoxylin and eosin (HE)-stained specimens obtained by transcervical needle biopsies from 331 patients with smooth muscle tumors and high intensity regions on $\mathrm{T} 1$ weighted images (WI) and/or T2WI from magnetic resonance imaging (MRI) scans were retrospectively examined. From a total of 10 patients with moderate or severe cytological atypia, 4 exhibited smooth muscle tumors of uncertain malignant potential and 6 exhibited leiomyosarcomas. The final diagnosis in 3 patients with $\geq 10$ mitotic figures/high-power field was leiomyosarcoma. A total of 5 patients with coagulative tumor cell necrosis exhibited final diagnoses of leiomyosarcoma. Patients without cytological atypia, mitotic figures or coagulative tumor cell necrosis were not diagnosed with either leiomyosarcomas or smooth muscle tumors of uncertain malignant potential. The present study revealed that laparoscopic surgery is safe when HE-stained specimens obtained by transcervical needle biopsy from areas of high intensity on an MRI scan are negative for all three criteria assessed-cytological atypia, mitotic figures and coagulative tumor cell necrosis.
\end{abstract}

Correspondence to: Dr Makoto Murakami, Department of Gynecology, Osaka City University, 1-5-7 Asahimachi, Abeno-ku, Osaka 5458586, Japan

E-mail: makoto19840415@yahoo.co.jp

Abbreviations: MRI, magnetic resonance imaging; HE, hematoxylin and eosin

Key words: needle biopsy, laparoscopic surgery, uterine smooth muscle tumors, histopathologically safe, leiomyosarcoma

\section{Introduction}

Uterine leiomyoma is the most common type of gynecological tumor, and is estimated to be present in $20-30 \%$ of women aged 30 and $40 \%$ of women aged $>40$. Uterine leiomyoma is more frequent in multiparous compared with in nulliparous women. Since laparoscopic surgery was introduced in 1979, it has increasingly replaced abdominal and vaginal approaches to operative treatments of uterine leiomyoma (1). However, in April 2014, the US Food and Drug Administration (FDA) issued a statement warning against the use of laparoscopic power morcellators for the majority of females undergoing a hysterectomy or myomectomy for uterine smooth muscle tumors due to the risk of spreading the cancerous tissue intraperitoneally if the tumors were malignant (2). Since this warning was issued, North American gynecologists have been reluctant to perform laparoscopic surgery on these types of tumor (3). Methods for the preoperative diagnosis of uterine myometrial lesions is a topic of discussion for Japanese gynecologists (4). Magnetic resonance imaging (MRI) scans are the most useful type of method for establishing a preoperative diagnosis of smooth muscle tumors. However, MRIs may be inaccurate when used as the sole diagnostic method. Since 1994, transcervical needle biopsies have been performed to differentiate between uterine leiomyomas and leiomyosarcomas (5).

In the present study, 331 patients with uterine smooth muscle tumors who underwent transcervical needle biopsies and possessed known subsequent outcomes were retrospectively reviewed. The aim of the present study was to assess the safety of laparoscopic excision of these types of tumors subsequent to transcervical needle biopsy and to evaluate the association between cytological atypia, mitotic activity and the presence of coagulative tumor cell necrosis in biopsy specimens and final diagnoses.

\section{Materials and methods}

Patients. Between June 1994 and May 2015, transcervical needle biopsies were performed in 626 patients in Osaka City University (Osaka, Japan) with written informed consent provided, subsequent to the necessity, risks and limitations of the diagnostic accuracy of this procedure having been explained. Outcomes 
were assessed in the 331 of the 626 patients who exhibited smooth muscle tumors and high intensity regions on T1 weighted images (T1WI) and/or T2 WI MRI scans. Of the 331 patients, the final diagnoses were: Leiomyosarcoma in 10 patients; smooth muscle tumor of uncertain malignant potential in 7 patients; and leiomyoma in 314 patients. All cases of leiomyosarcoma met the Stanford criteria of Bell et al (6). Smooth muscle tumors of uncertain malignant potential were defined as those that could not be classified reliably as benign or malignant on the basis of generally applied criteria (7). Due to the fact that none of the tumors initially diagnosed as smooth muscle tumors of uncertain malignant potential in the present study met the Stanford criteria for leiomyosarcoma, but they did exhibit moderate to severe atypia, they were classified as atypical leiomyomas. In the present study, 76 of the 314 leiomyomas were diagnosed using specimens obtained from surgery performed subsequent to needle biopsy, whereas the remaining 238 leiomyomas were diagnosed using biopsy specimens and these latter patients did not undergo surgery. As the present study was a retrospective study of records from patients, the Ethics Committee of Osaka City University (Osaka, Japan) waived the requirement for informed consent.

The retrospective assessment of hematoxylin and eosin (HE)-stained transcervical needle biopsy specimens from 331 patients determined whether the Stanford criteria of Bell et al (6): The presence of cytological atypia, the number of mitotic figures and the presence of coagulative tumor cell necrosis, which may be used to construct an algorithm to perform laparoscopic surgery with histopathological safety. Patients with any combination of cytological atypia, mitotic figures and coagulative tumor cell necrosis were identified and classified according to their final diagnoses, as presented in Fig. 1. Patients exhibiting cytological atypia were allocated into 4 groups based on the degree of atypia and the ratio of these groups was plotted, as presented in Fig. 2. The procedure was repeated and used to construct plots based on the number of mitotic figures and the presence of coagulative tumor cell necrosis, as presented in Figs. 3 and 4. A Mann-Whitney U test was performed to ascertain the significance of the mitotic counts, as presented in Fig. 3. $\mathrm{P}<0.05$ was considered to indicate a statistically significant difference.

Needle biopsy. The transcervical needle biopsies of smooth muscle tumors were performed using an automatic $25-\mathrm{cm}$ long, 16-gauge, 17-mm notch cutting needle and a $20-\mathrm{cm}$ long straight stainless steel guide pipe with a $4 \mathrm{~mm}$ maximum external dimension and a $3 \mathrm{~mm}$ maximum internal dimension (Honest Medical Co., Ltd., Tokyo, Japan), under transabdominal guidance using a Pro-Mag Ultra Biopsy (Argon Medical Devices, Plano, TX, USA) or a Pro-Mag 2.2 biopsy system (Argon Medical Devices). Analgesics (25 or $50 \mathrm{mg}$ diclofenac sodium suppositories in almost all cases) were administered prior to biopsy as required. The patients were then placed in the lithotomy position and a guide pipe was inserted transcervically into the uterine cavity. The operator manipulated the uterine corpus and guided the pipe using transabdominal ultrasonic guidance, to align the pipe with the lesion to be biopsied. The biopsy needle was then inserted through the guide pipe into the uterine tumor. Subsequent to confirming that the tip of the needle was within the lesion, the biopsy gun (Argon Medical Devices) was used to obtain a tissue sample (5). To minimize sampling error, 3 biopsy specimens/patient were obtained.

Histopathological analysis. A total of 3 biopsy specimens from each patient were evaluated using hematoxylin \& eosin staining. Specimens were fixed in $10 \%$ formalin at $20^{\circ} \mathrm{C}$ for $24 \mathrm{~h}$, paraffin-embedded and cut into $4 \mu \mathrm{m}$ sections. Samples were deparaffinized using $99 \%$ xylene and alcohol dehydration of graded series from 100 to $70 \%$ ethanol. Finally, samples were stained with $0.15 \%$ hematoxylin solution diluted 4 times for $4.5 \mathrm{~min}$ at $20^{\circ} \mathrm{C}$, followed by $0.33 \%$ Eosin for $4 \mathrm{~min}$ at $20^{\circ} \mathrm{C}$. The degree of cytological atypia was recorded as follows: Severe atypia, detectable at low magnification (magnification, $\mathrm{x} 4$, light microscope); moderate atypia (magnification, $\mathrm{x} 10-20$ ); mild atypia (magnification, $\mathrm{x} 40$ ) and no atypia. The mitotic figures were counted under a light microscope in all high-power fields for the 3 specimens using x40 objective lens. Levels of coagulative tumor cell necrosis were recorded as follows: Positive, which was confirmed by presence of ghost cells and abrupt transition, as presented in Fig. 5; suspected, which was defined as the presence of ghost cells with unclear abrupt transition; and negative, which was confirmed by evidence of hyalinization only (5). The patients with particular variants of leiomyoma, such as epithelioid leiomyoma or lipoleiomyoma, were excluded from additional analyses.

\section{Results}

A total of 33 patients exhibited cytological atypia in their biopsy specimens; 7 with a diagnosis of leiomyosarcoma, 7 with a smooth muscle tumor of uncertain malignant potential and 19 with a diagnosis of leiomyoma. Thus, the final diagnosis was leiomyoma in $~ 58 \%$ (19/33) of the patients with cytological atypia, as presented in Fig. 1. Of the 10 patients with moderate or severe cytological atypia, 4 exhibited smooth muscle tumors of uncertain malignant potential and 6 exhibited leiomyosarcomas. No cytological atypia was found in 298 patients, $99 \%$ of whom exhibited leiomyomas. However, the final diagnosis of 3 patients with no cytological atypia in their biopsy specimens, was leiomyosarcoma, as presented in Fig. 2.

A total of 32 patients exhibited between 1 and 9 mitotic figures in all high-power fields: 5 patients with leiomyosarcoma, 1 patient with a smooth muscle tumor of uncertain malignant potential, and 26 with leiomyoma. In 3 of the patients with $\geq 10$ mitotic figures in all high-power fields, the final diagnosis was leiomyosarcoma, as presented in Fig. 1. A total of 26 patients with leiomyoma that exhibited mitotic figures on biopsy specimens possessed $<10$ mitotic figures in all high-power fields. The median number of mitotic figures differed significantly between incidences of leiomyosarcoma and smooth muscle tumors of uncertain malignant potential, and between leiomyosarcoma and leiomyoma, as presented in Fig. 3.

A total of 34 patients exhibited a confirmed positive result or suspected coagulative tumor cell necrosis, 8 with leiomyosarcoma, 1 with a smooth muscle tumor of uncertain malignant potential, and 25 with leiomyoma, as presented in Fig. 1. A total of 5 patients with coagulative tumor cell necrosis had final diagnoses of leiomyosarcoma. Coagulative tumor cell necrosis was not exhibited in 297 patients, $97 \%$ of whom exhibited leiomyomas. Of the patients with suspected 


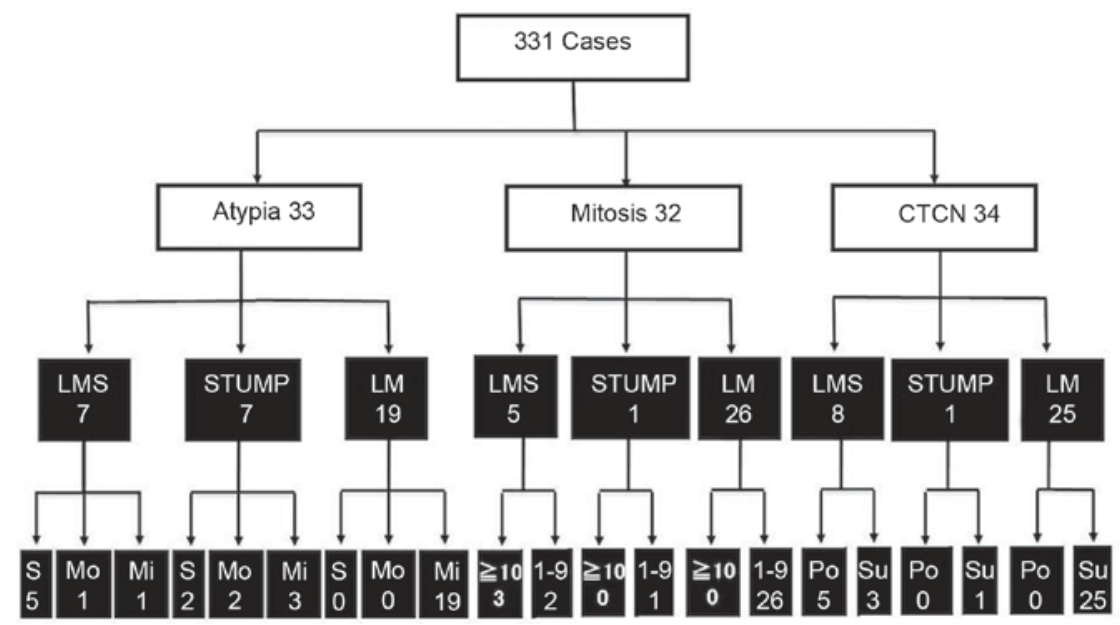

Figure 1. Tumors identified as exhibiting cytological atypia and/or mitotic figures and/or coagulative tumor cell necrosis classified according to final diagnoses. The values indicate the number of cases in each category. S, severe; Mo, moderate; Mi, mild; Po, positive; Su, suspicious; LMS, leiomyosarcoma; STUMP, smooth muscle tumor of uncertain malignant potential; LM, leiomyoma; CTCN, coagulative tumor cell necrosis.

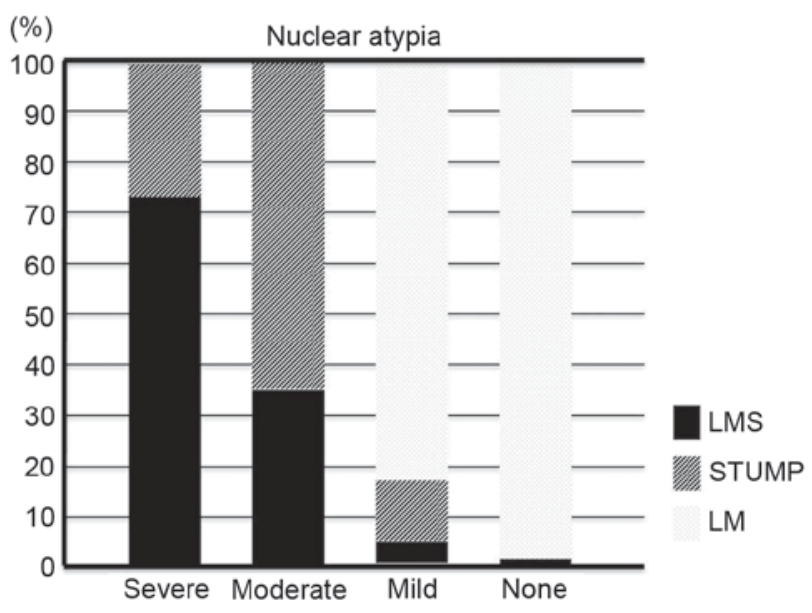

Figure 2. Allocation of tumors with cytological atypia into 4 groups based on the degree of atypia shown as \% of diagnosis in each group. LMS, leiomyosarcoma; STUMP, smooth muscle tumor of uncertain malignant potential; LM, leiomyoma.

coagulative tumor cell necrosis, $86 \%$ exhibited leiomyomas and $10 \%$ exhibited leiomyosarcomas, as presented in Fig. 4.

In the present study, no patients without cytological atypia, mitotic figures or coagulative tumor cell necrosis were diagnosed with either leiomyosarcomas or smooth muscle tumors of uncertain malignant potential. The 238 patients with leiomyomas who did not undergo surgery were followed up for $>2$ years, during which time they exhibited no symptoms of leiomyosarcoma, such as a rapid increase in the size of lesions or an increase in lesion size subsequent to menopause.

\section{Discussion}

A previous study estimated that $1 / 350$ women who undergo a hysterectomy develop malignant uterine tumors (2). Since then, North American gynecologists have been reluctant to perform laparoscopic surgery for uterine smooth muscle tumors (3), and methods for the preoperative diagnosis of uterine myometrial lesions is a topic of debate amongst Japanese gynecologists (4).

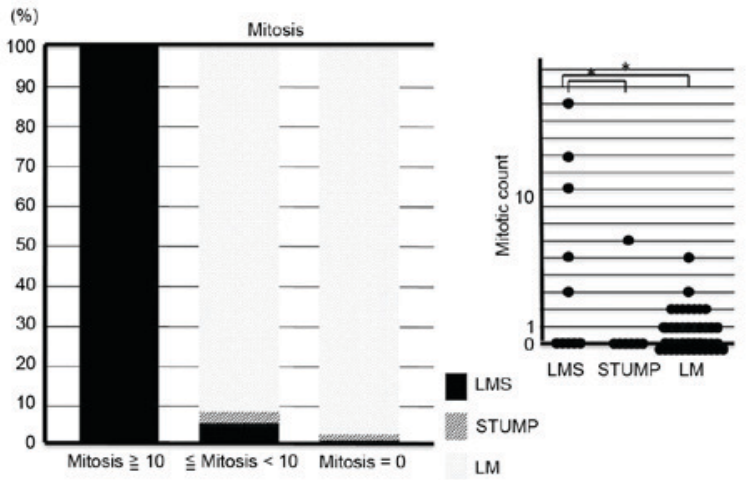

Figure 3. Allocation of tumors with mitotic figures to 3 groups based on mitotic count shown as the percent of diagnoses in each group. The Mann-Whitney U test was used to ascertain the significances of differences in mitotic count. LMS, leiomyosarcoma; STUMP, smooth muscle tumor of uncertain malignant potential; LM, leiomyoma. ${ }^{*} \mathrm{P}<0.05$.

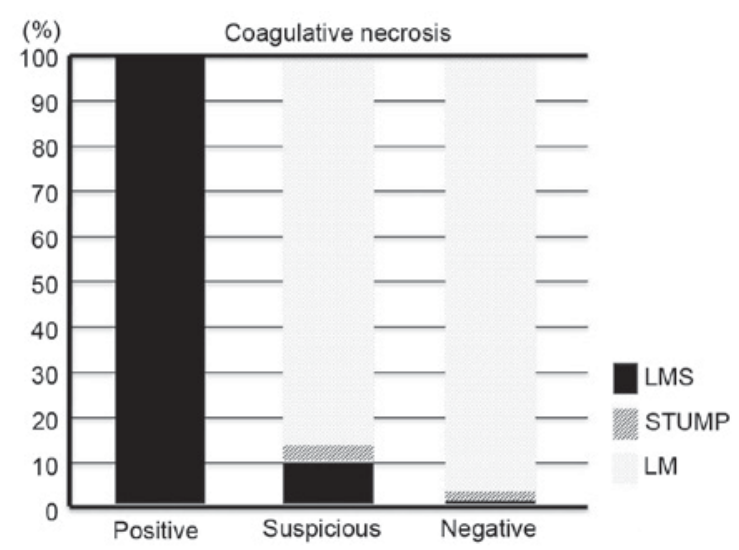

Figure 4. Allocation of tumors with coagulative tumor cell necrosis to 3 groups based on the presence or absence of coagulative tumor cell necrosis shown as \% of diagnosis in each group. LMS, leiomyosarcoma; STUMP, smooth muscle tumor of uncertain malignant potential; LM, leiomyoma.

MRI scans are the most useful technique of preoperatively diagnosing smooth muscle tumors. Leiomyosarcomas typically exhibit a high intensity with T2WI, which reflects the growth 


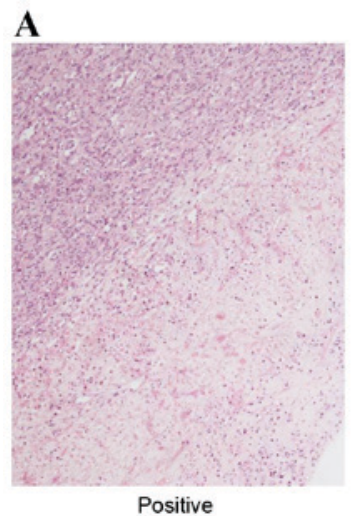

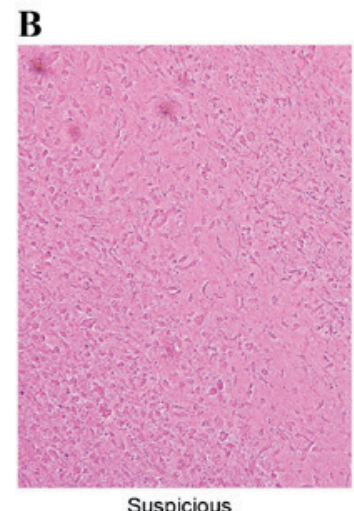

Suspicious

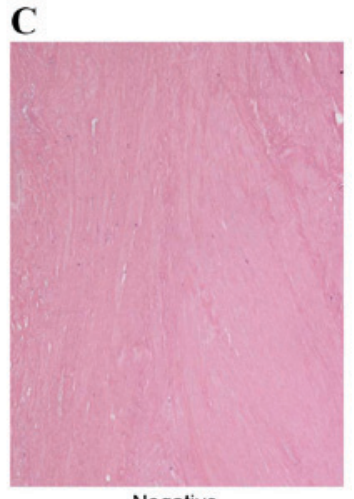

Negative

Figure 5. (A) CTCN positive: CTCN was characterized by an abrupt transition from viable cells to ghost cells without an interposed zone of granulation tissue or hyalinized tissue between the viable and necrotic cells. Magnification, x4. (B) CTCN suspicious: Presence of ghost cells with an unclear abrupt transition. Magnification, x20. (C) CTCN negative: Hyalinization only. Magnification, x4. CTCN, coagulative tumor cell necrosis.

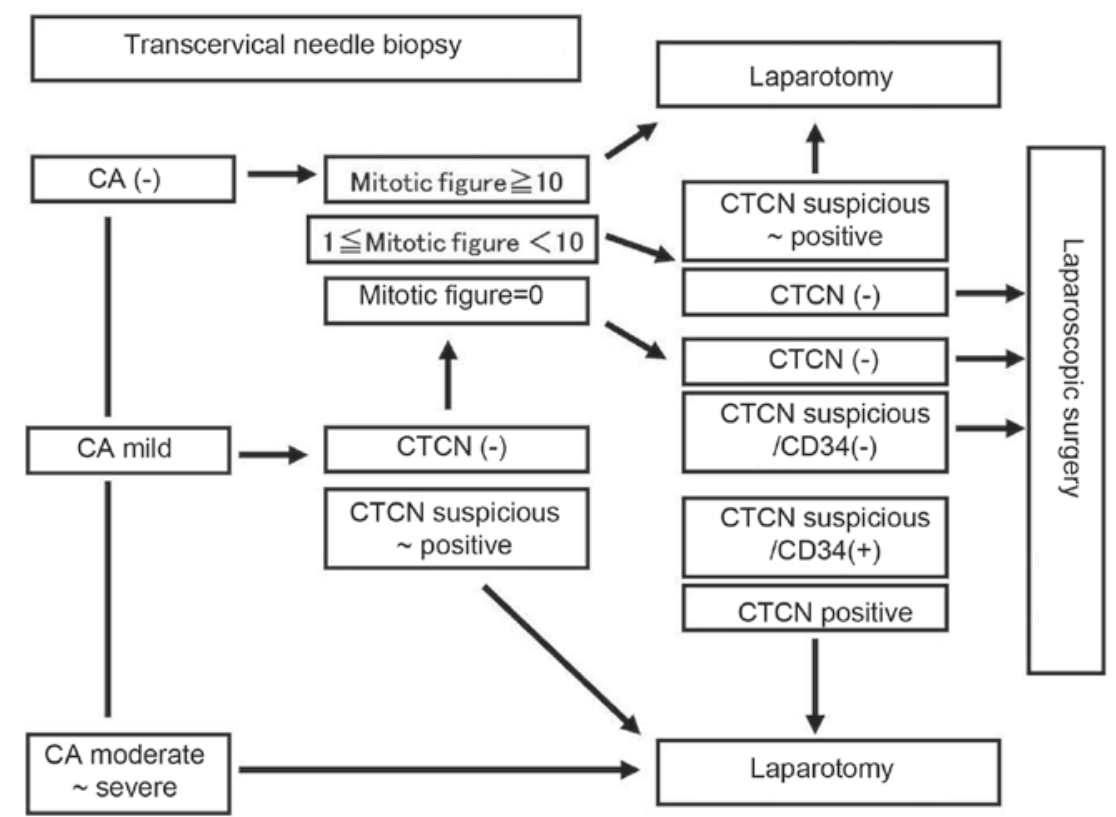

Figure 6. Proposed algorithm for indications for laparoscopic surgery by transcervical needle biopsy. CD34(+), $>10$ CD34-stained vessels in necrotic areas; CD34(-), <10 CD34-positive vessels in necrotic areas. CD, cluster of differentiation; CTCN, coagulative tumor cell necrosis; CA, cytological atypia.

of cells, and high intensity with T1 and T2WI, which reflects hemorrhage. However, as some uterine leiomyomas exhibit high intensity on T1 and/or T2WI images and some leiomyosarcomas do not exhibit clear high intensity region on T1WI images, smooth muscle tumors cannot be reliably diagnosed using MRI scans alone (5). Since 1994, transcervical needle biopsies have been performed in addition to MRIs, to accurately exclude the diagnosis of malignant diseases such as leiomyosarcoma. To determine whether transcervical needle biopsies are useful for performing laparoscopic surgeries for uterine smooth muscle tumors with histopathological safety, the present study retrospectively assessed 331 patients with smooth muscle tumors who had undergone the aforementioned procedure.

Of the 331 patients in the present study, all 250 that did not exhibit the 3 Stanford criteria: Cytological atypia, mitotic figures and coagulative tumor cell necrosis, and exhibited high intensity regions on MRI scans were diagnosed with leiomyoma. Thus, laparoscopic surgery is histopathologically safe when these 3 factors are negative in the transcervical needle biopsy specimens. However, if moderate or severe cytological atypia is found in transcervical needle biopsy specimens, the tumor is likely to be a smooth muscle tumor of uncertain malignant potential or leiomyosarcoma, and laparoscopic surgery is therefore not recommended. Additionally, in the present study, leiomyosarcoma was the final diagnosis in 1 patient with mild cytological atypia and in 3 patients with no cytological atypia. The specimens of these patients (1 patient with mild cytological atypia and 3 patients with no cytological atypia) comprised mainly necrotic areas with few viable cells. As biopsies target the parts of tumors with abnormal MRI findings, necrotic tissue is often obtained in patients with leiomyosarcoma. When a specimen contains areas with suspected or positive coagulative tumor cell necrosis, laparoscopic surgery is not recommended, due to the limited number of cells available from viable areas leading to an underestimation of the degree of cytological atypia. 
Laparoscopic surgery is not recommended in patients with tumors that exhibit $\geq 10$ mitotic figures in all fields examined, as such tumors are likely to be leiomyosarcomas. The patients with biopsy specimens that contained 1-9 mitotic figures received final diagnoses of leiomyosarcoma. However, in all such cases, the biopsy specimens exhibited areas with suspected or positive coagulative tumor cell necrosis regions and a limited number of viable cells. Therefore, when mitotic figures in transcervical needle biopsy specimens containing necrotic regions are identified, laparoscopic surgery is considered contraindicated in these patients for the same reasons as it is in patients with cytological atypia, mainly due to the limited number of cells available from viable areas leading to an underestimation of the degree of mitotic figures.

As the possibility of leiomyosarcoma is high when coagulative tumor cell necrosis is present, laparoscopic surgery is not recommended in such patients. Of patients with suspected coagulative tumor cell necrosis, $\sim 10 \%$ exhibit leiomyosarcoma. Patients with a final diagnosis that is not leiomyosarcoma but who exhibit suspected coagulative tumor cell necrosis, also occasionally exhibit hyaline necrosis in leiomyoma or smooth muscle tumors of uncertain malignant potential. The presence of hyaline necrosis does not contraindicate laparoscopic surgery. However, it may be difficult to differentiate between coagulative tumor cell necrosis and hyaline necrosis on HE-stained specimens. We previously reported that coagulative tumor cell necrosis and hyaline necrosis contain significantly different numbers of anti-cluster of differentiation (CD)34 antibody-positive vessels. Vessels in the areas of coagulative tumor cell necrosis appear to retain immunostaining by the anti-CD34 antibody. In the present study, CD34-stained vessels were counted for each specimen at high-power fields (magnification, $\mathrm{x} 40$ ) and the highest number of positive vessels was recorded. We found that $\sim 80 \%$ of leiomyosarcomas exhibit $>10$ CD34-stained vessels in necrotic areas and that the highest combined sensitivity and specificity is achieved by setting the cut-off score at 10 CD34-stained vessels (8). None of the smooth muscle tumors of uncertain malignant potential examined in the hospital of Osaka City University have exhibited coagulative tumor cell necrosis, thus, this combination is likely to be rare. Laparoscopic surgery is considered acceptable in patients with biopsies that are negative for coagulative tumor cell necrosis, or in patients with <10 CD34-positive vessels in lesions with suspected coagulative tumor cell necrosis. Based on the data obtained in the present study, the proposed algorithm for the indications for laparoscopic surgery is presented in Fig. 6.

Laparoscopic surgery for smooth muscle tumors is considered histopathologically safe when HE-stained specimens, obtained by transcervical needle biopsy of areas in tumors exhibiting high intensity on MRI scans, are negative for all 3 of the following criteria: Cytological atypia, mitotic figures and coagulative tumor cell necrosis. However, if any combination of moderate or severe cytological atypia, $>10$ mitotic figures and coagulative tumor cell necrosis is identified in the transcervical needle biopsy specimens, laparoscopic surgery is not recommended.

\section{Acknowledgements}

Not applicable.

\section{Funding}

No funding was received.

\section{Availability of data and materials}

All data generated or analyzed during this study are included in this published article.

\section{Authors' contributions}

$\mathrm{MMu}$ designed the study and wrote the initial draft of the manuscript. TI, MK, MMa and NK contributed to analysis and interpretation of data, and assisted in the preparation of the manuscript. TF and TS contributed to data collection and interpretation and critically reviewed the manuscript. All authors approved the final version of the manuscript, and agreed to be accountable for all aspects of the work in ensuring that questions related to the accuracy or integrity of any part of the work are appropriately investigated and resolved.

\section{Ethics approval and consent to participate}

As the present study was retrospective, the Ethics Committee of Osaka City University (Osaka, Japan) waived the requirement for informed consent.

\section{Consent for publication}

Not applicable.

\section{Competing interests}

The authors declare that they have no competing interests.

\section{References}

1. Semm K: New methods of pelviscopy (gynecologic laparoscopy) for myomectomy, ovariectomy, tubectomy and adrenectomy. Endoscopy 11: 85-93, 1979.

2. U.S. Food and drug administration. Laparoscopic uterine power morcellation in hysterectomy and myomectomy: FDA safety communication. http://www.fda.gov/medicaldevices/safety/alertsandnotices/ucm393576.htm. Accessed November, 2014.

3. Ton R, Kilic GS and Phelps JY: A medical-legal review of power morcellation in the face of the recent FDA warning and litigation. J Minim Invasive Gynecol 22: 564-572, 2015.

4. Tanaka O, Nishida M, Tsunoda H, Okamoto Y and Yoshikawa H: Smooth muscle tumors of uncertain malignant potential and leiomyosarcomas of the uterus: MR findings. J Magn Reson Imaging 20: 998-1007, 2004.

5. Kawamura N, Ichimura T, Ito F, Shibata S, Takahashi $K$, Tsujimura A, Ishiko O, Haba T, Wakasa K and Ogita S: Transcervical needle biopsy for the differential diagnosis between uterine sarcoma and leiomyoma. Cancer 94: 1713-1720, 2002.

6. Bell SW, Kempson RL and Hendrickson MR: Problematic uterine smooth muscle neoplasms. A clinicopathologic study of 213 cases. Am J Surg Pathol 18: 535-538, 1994.

7. International Agency for Research on Cancer (IARC): WHO Classification of Tumours of Female Reproductive Organs. Kurman RJ, Carcangiu ML, Herrington CS and Young RH (eds). 4th edition. IARC, Lyon, pp238-239, 2014.

8. Yoshida C, Ichimura T, Kawamura N, Nakano A, Kasai M, Sumi T and Ishiko O: A scoring system for histopathologic and immunohistochemical evaluations of uterine leiomyosarcomas. Oncol Rep 22: 725-731, 2009. 